\title{
A New Co-Crystal of Synthetic Drug Rosiglitazone with Natural Medicine Berberine: Preparation, Crystal Structures, and Dissolution
}

\author{
Xiaoshu Guan ${ }^{1,+}$, Lan Jiang ${ }^{2,+}$, Linhong Cai ${ }^{1}$, Li Zhang ${ }^{1}(\mathbb{D})$ and Xiangnan Hu ${ }^{1, *(D)}$ \\ 1 Department of Medicinal Chemistry, School of Pharmacy, Chongqing Medical University, \\ Chongqing 400016, China; 17082343045@163.com (X.G.); cailinhong123@163.com (L.C.); \\ zhangli3357@163.com (L.Z.) \\ 2 College of Environment and Resources, Chongqing Technology and Business University, \\ Chongqing 400016, China; jianglanlucky@163.com \\ * Correspondence: huxiangnan@cqmu.edu.cn; Tel.: +86-133-0836-0519 \\ + Lan Jiang is co-first author, supervised by Xiaoshu Guan.
}

Received: 5 August 2020; Accepted: 15 September 2020; Published: 18 September 2020

check for updates

\begin{abstract}
A co-crystal of rosiglitazone (Rsg) with berberine (Bbr), Rsg-Bbr, was prepared by the solvent evaporation method and characterized. The results showed that the electrostatic attraction existed between the nitrogen anion of rosiglitazone and the quaternary ammonium cation of berberine, and C-H...O hydrogen bonds were formed between Rsg and Bbr. In the crystal structure, rosiglitazone molecules stack into a supramolecular layer through $\pi-\pi$ interactions while $\pi-\pi$ interactions between berberine cations also result in a similar layer. The co-crystal presented a low moisture adsorption curve in the range of $0-95 \%$ relative humidity values at $25^{\circ} \mathrm{C}$. The improved dissolution rate of rosiglitazone in $\mathrm{pH}=6.8$ buffer solution could be achieved after forming co-crystal.
\end{abstract}

Keywords: co-crystal; rosiglitazone; berberine; electrostatic attraction; moisture adsorption; dissolution rate

\section{Introduction}

Although the co-crystal has a history of more than 100 years, it has been used in the field of medicine in the last two decades [1]. The new drug co-crystal is formed by an active pharmaceutical ingredient (API) and another or more cocrystal formers (CCFs) with the action of non-covalent bonds, which could improve many physical and chemical properties, such as solubility and stability $[2,3]$. It is worth noting that drug-drug co-crystals based on two APIs have attracted new attention in the pharmaceutical field recently [4-6]. This multidrug crystal is superior to traditional drug crystal because it is one drug that contains two APIs to achieve the effect of synergistic treatment for specific diseases [7]. Take an example, entresto, the first dual-acting angiotensin receptor-neprilysin inhibitor (ARNi) drug composed of sacubitril and valsartan, has the therapeutic advantages beyond monomer drugs $[8,9]$. Besides, the formation of reliable supramolecular synthons between two different chemical structures of APIs is essential for the rational design of drug-drug co-crystals [10].

Rosiglitazone (Rsg, Figure 1a), is a highly selective and effective agonist of a peroxisome proliferation-activated receptor- $\gamma(\operatorname{PPAR} \gamma)$, with controlling blood sugar by increasing insulin sensitivity [11,12]. It serves as a first-line thiazolidinedione-containing drug for type 2 diabetes mellitus (T2DM) [13]. Unfortunately, due to the low solubility and high permeability of the molecule itself, Rsg is ranked as a class II molecule by the Biopharmaceutical Classification System (BCS) [14]. The poor solubility of rosiglitazone may affect its effectiveness in the body. Therefore, attempting 
to tackle the solubility problem and other undesirable properties of Rsg by co-crystallization is worth exploring.

Berberine(Bbr) is one of the most important bioactive isoquinoline alkaloids from the well-known Traditional Chinese Medicine called "Huang Lian", which has been used in China for over 1000 years [15]. Bbr exists in nature mainly in the form of quaternary ammonium salt, with its chloride salt (Figure $1 \mathrm{~b}$ ) used in the clinical medication. In the research of efficacy, it was found to have various therapeutic effects such as anti-inflammatory [16], inhibiting glucose absorption [17], regulating intestinal flora [18] and protecting intestinal mucosal barrier [19] activities. Interestingly, Bbr has been used to treat type 2 diabetes in recent years [20]. It could increase insulin receptor expression, promote the release and secretion of insulin, and increase the glucose consumption of liver cells [21,22], etc. Due to its feasible hypoglycemic effect, berberine was used in combination with other hypoglycemic drugs to treat type 2 diabetes in clinical drug research [23]. Moreover, its chloride salt has good solubility in water [24], which meets the conditions of co-crystal formers.

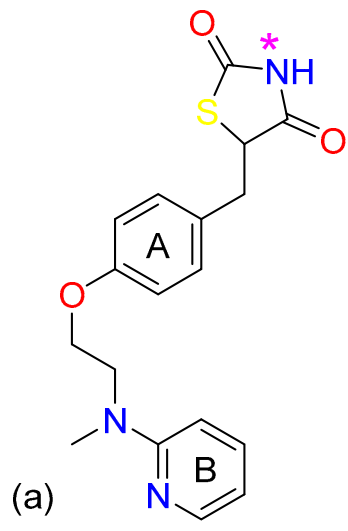

(b)

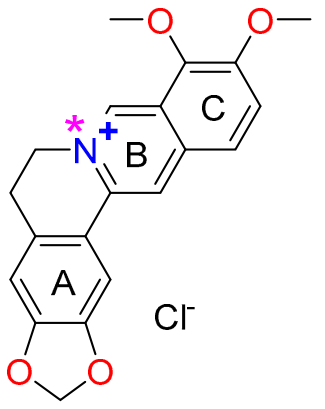

Figure 1. Chemical structures of the Rosiglitazone (a) and Berberine chloride (b) with the N-H and quaternary ammonium cation sites highlighted, respectively.

In this work, we seek to address the adverse properties of Rsg, such as solubility, by the technique of crystal engineering. A search of literature including the Cambridge Structure Database (CSD) found that the crystal form reports of rosiglitazone was mostly related to its acid salt forms, while the co-crystallization with other drugs was rarely reported. Therefore, it is worthy to investigate drug-drug co-crystals based on Rsg and another APIs. Because of the excellent therapeutic effect on type 2 diabetes and the water solubility of its chloride, we decided to choose the Bbr to be a co-crystal former of rosiglitazone.

By analyzing the structure of two compounds, we found that the N-H bond on the thiazolidinedione of rosiglitazone easily loses protons to become a nitrogen anion with the influence of two adjacent carbonyl groups. It should be noted that berberine, a quaternary ammonium cation, contains a positive charge without acquiring a proton, unlike amine cations. Considering the compound charge stability, the cationic nature of berberine requires the presence of charge equivalent anion to maintain charge neutrality. Thus, we speculate whether the nitrogen anion on the thiazolidinedione ring can be substituted for the chloride ion in berberine chloride to allow rosiglitazone and berberine to form a novel adduct through non-covalent bonds. In this paper, the co-crystallization of rosiglitazone and berberine was carried out by the solvent volatilization method. As a result, the co-crystal Rsg-Bbr has successfully been prepared. Its structure was characterized by Fourier-transform infrared (FT-IR), powder X-ray powder diffraction (PXRD), thermogravimetry (TGA), differential scanning calorimetry (DSC), single crystal X-ray diffraction (SCXRD), and polarized optical microscopy (POM). The dissolution rate and hygroscopicity of the co-crystal were also explored. 


\section{Results and Discussion}

\subsection{The Characterization Analysis of the Co-Crystal Structure}

With the anion exchange reaction and the simple method of solvent evaporation, Rsg-Bbr was successfully prepared in the methanol within five days. The FT-IR and PXRD were used for the preliminary detection of the difference between Rsg-Bbr and two raw materials.

Infrared spectroscopy is a simple and rapid test method in the co-crystal screening experiment, it can preliminarily identify the difference between the co-crystal and two materials by comparing with individual components. In the infrared spectra of the Rsg-Met (Figure 2), the N-H stretching vibration $\left(3379 \mathrm{~cm}^{-1}\right.$ ) of Rsg disappeared, which was influenced by the removal of the $\mathrm{H}$ atom of the amino group makes nitrogen become anionic. The thiolactone $\mathrm{C}=\mathrm{O}$ vibration $\left(1693 \mathrm{~cm}^{-1}\right) \mathrm{of} R \mathrm{sg}$ moved to low-frequency and displayed a novel absorption peak at $1681 \mathrm{~cm}^{-1}$, which was influenced by the formation of $\mathrm{N}$ anions, and the intermolecular hydrogen bonds. Furthermore, compared with the corresponding groups in the pure Bbr material, the $C=N$ vibration peak $\left(1634 \mathrm{~cm}^{-1}\right) \mathrm{moved}$ to low-frequency and showed a novel absorption peak at $1598 \mathrm{~cm}^{-1}$, which was affected by the electrostatic interaction. The $C=C$ of benzene rings stretching vibration peak $\left(1600 \mathrm{~cm}^{-1}\right)$ moved to low-frequency with the influence of the $\pi-\pi$ effect between molecular layers and displayed a novel absorption peak at $1562 \mathrm{~cm}^{-1}$. These results indicated that the intermolecular interaction could cause some subtle changes in the molecular structure.

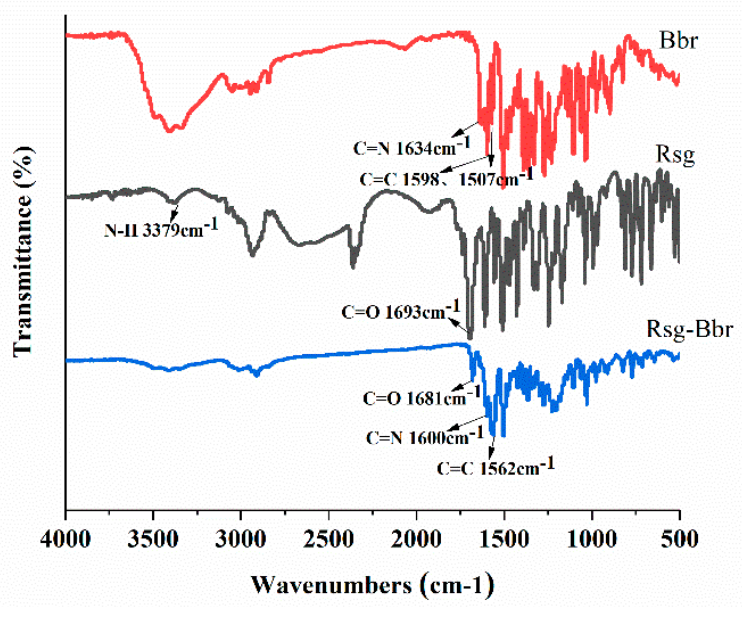

Figure 2. IR spectral data for Bbr, Rsg, and Rsg-Bbr.

Powder X-ray diffraction pattern of Rsg-Bbr showed a new characteristic peak by comparing with two monomer components (Figure 3). The characteristic peaks (two theta in degree) of the reactants and product were as following, Rsg (15.2, 15.6, 17.4, 18.2, 20.1, 22.4, 32.2), Bbr (6.5, 9.18, 10.71, 13.14, $25.15,25.56,26.71)$ and Rsg-Bbr $(16.3,20.9,25.01,25.5,30.6,30.8)$. Additionally, the experimental PXRD patterns of Rsg-Bbr well matched the simulated PXRD patterns of corresponding single crystal structure. This confirmed the phase purity of the bulk powders.

The data of this co-crystal measured by single crystal X-ray diffraction (SCXRD) experiment further confirmed the formation of Rsg-Bbr. The corresponding crystallographic data and refinement details were summarized in Table 1 . The crystal structure of Rsg-Bbr was in the triclinic space group P-1: $a=7.4411(4) \AA, b=13.3185(6) \AA, c=18.8457(10) \AA, \alpha=98.950(4)^{\circ}, \beta=98.400(4)^{\circ}, \gamma=101.178(4)^{\circ}$, $\mathrm{Z}=2$, and the final $\mathrm{R}_{1}$ was $0.0584(\mathrm{I}>2 \sigma(\mathrm{I}))$ and $w \mathrm{R}_{2}$ was 0.1833 (all data). The asymmetric unit of the solid is shown in Figure 4, it contained one molecule of Rsg, one molecule of Bbr, and one molecule of methanol. 


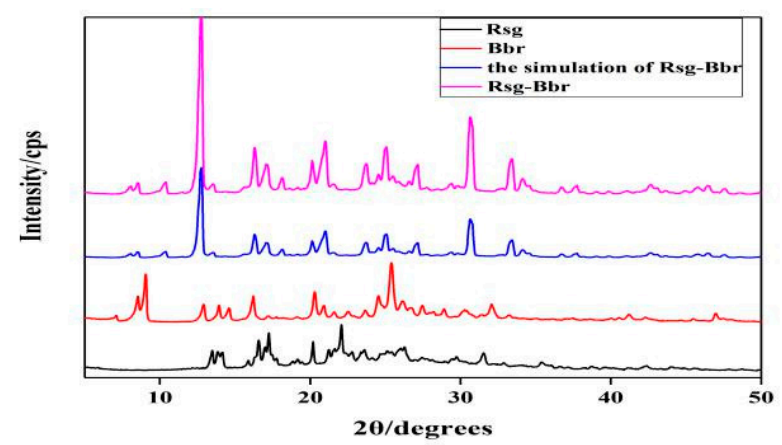

Figure 3. X-ray powder diffractograms of Rsg-Bbr, Rsg and Bbr.

Table 1. Crystal Data and Structural Refinement for Rsg-Bbr.

\begin{tabular}{|c|c|}
\hline Empirical Formula & $\mathrm{C}_{39} \mathrm{H}_{40} \mathrm{~N}_{4} \mathrm{O}_{8} \mathrm{~S}$ \\
\hline molecular weight & 724.81 \\
\hline Crystal size $/ \mathrm{mm}^{3}$ & $0.22 \times 0.15 \times 0.12$ \\
\hline Crystal system & triclinic \\
\hline Space group & $P-1$ \\
\hline $\mathrm{a} / \AA$ & $7.4411(4)$ \\
\hline $\mathrm{b} / \AA$ & $13.3185(6)$ \\
\hline$c / \AA$ & $18.8457(10)$ \\
\hline$\alpha /^{\circ}$ & $98.950(4)$ \\
\hline$\beta /{ }^{\circ}$ & $98.400(4)$ \\
\hline$\gamma /{ }^{\circ}$ & $101.178(4)$ \\
\hline $\mathrm{V} / \AA^{3}$ & $1779.75(16)$ \\
\hline Z & 2 \\
\hline$\rho_{\text {calc }} \mathrm{g} / \mathrm{cm}^{3}$ & 1.353 \\
\hline$\mu / \mathrm{mm}^{-1}$ & 1.307 \\
\hline $\mathrm{F}(000)$ & 764.0 \\
\hline $2 \theta$ range for data collection $/^{\circ}$ & 7.628 to 134.16 \\
\hline Index ranges & $-8 \leq \mathrm{h} \leq 8,-15 \leq \mathrm{k} \leq 10,-22 \leq 1 \leq 22$ \\
\hline Reflections collected & 12524 \\
\hline Independent reflections & $6335\left[R_{\text {int }}=0.0261, R_{\text {sigma }}=0.0361\right]$ \\
\hline Data/restraints/parameters & $6335 / 53 / 496$ \\
\hline Goodness-of-fit on $\mathrm{F}^{2}$ & 1.038 \\
\hline Final $R$ indexes $[\mathrm{I} \leq 2 \sigma(\mathrm{I})]$ & $\mathrm{R}_{1}=0.0584, \mathrm{wR}_{2}=0.1635$ \\
\hline Final R indexes [all data] & $\mathrm{R}_{1}=0.0771, \mathrm{wR}_{2}=0.1833$ \\
\hline Largest diff. peak/hole / e $\AA^{-3}$ & $0.40 /-0.26$ \\
\hline CCDC no. & 2007762 \\
\hline
\end{tabular}

However, only weak D-H...A hydrogen bonds (Table 2) were present to stabilize this structure. As shown in Table 2 and Figure 5, there existed obvious hydrogen-bonding interactions between Rsg and Bbr. Specifically, Bbr established the hydrogen bonding by the $11-\mathrm{H}$ atom and $14-\mathrm{H}$-atom on the B-pyridine ring with the lactam group and the thiolactone group of the Rsg molecule, respectively $\mathrm{C}(11)-\mathrm{H}(11) \cdots \mathrm{O}(6)$ and $\mathrm{C}(14)-\mathrm{H}(14) \cdots \mathrm{O}(5)$. 5-carbonyl group of Rsg was involved in intramolecular hydrogen-bonding interactions with the $\mathrm{H}_{-} \mathrm{D}^{\wedge} \mathrm{b}$ atom on 24 -methlene group $\left[\mathrm{C}\left(24 \mathrm{~A}^{\wedge} \mathrm{b}\right)-\mathrm{H}\left(24 \mathrm{D}^{\wedge} \mathrm{b}\right) \cdots \mathrm{O}(5)\right]$. As a solvent, methanol interacted with rosiglitazone ion and berberine ion by forming hydrogen bonds $\mathrm{O}\left(8 \mathrm{~A}^{\wedge} \mathrm{b}\right)-\mathrm{H}\left(8 \mathrm{AA}^{\wedge} \mathrm{b}\right) \cdots \mathrm{N}\left(4 \mathrm{~A}^{\wedge} \mathrm{b}\right)$ and $\mathrm{C}(7)-\mathrm{H}(7) \cdots \mathrm{O}\left(8^{\wedge} \mathrm{a}\right)$, respectively. In addition, the electrostatic attraction between the nitrogen anion of rosiglitazone and the quaternary ammonium cation of berberine made a contribution to stabilize the crystal structure. 


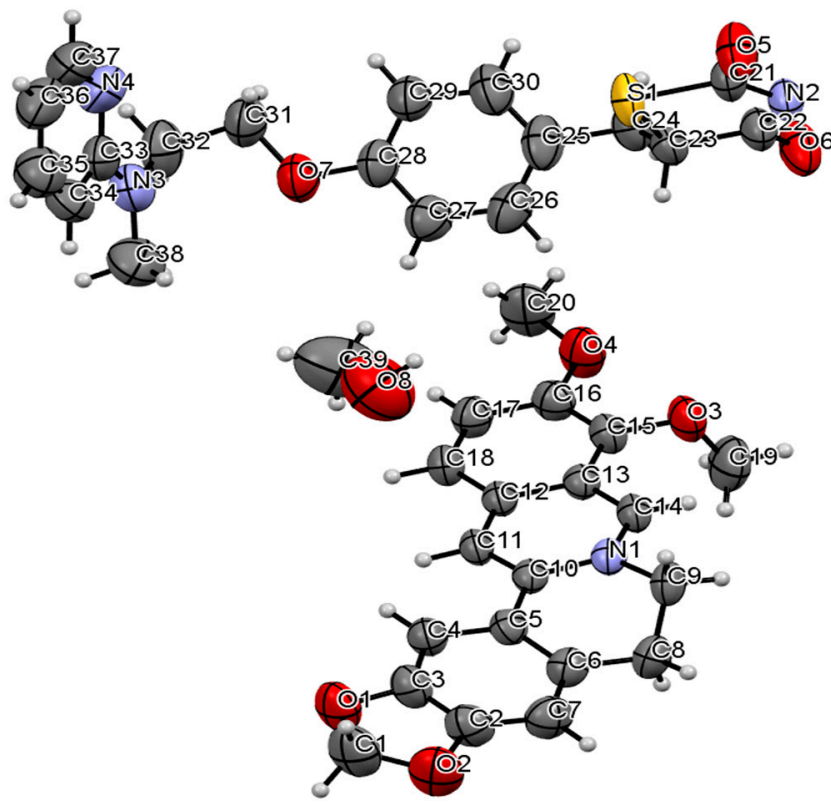

Figure 4. The ORTEP diagram of Rsg-Bbr.

Table 2. Hydrogen bonding table for the co-crystal Rsg-Bbr.

\begin{tabular}{|c|c|c|c|c|}
\hline $\mathrm{D}-\mathrm{H} \cdots \mathrm{A}$ & $\mathrm{d}(\mathrm{D}-\mathrm{H}) / \AA$ & $\mathrm{d}(\mathbf{H} \cdots \mathbf{A}) / \AA$ & $\mathrm{d}(\mathrm{D} \cdots \mathrm{A}) / \AA$ & D-H-A/ ${ }^{\circ}$ \\
\hline $\mathrm{C}(11)-\mathrm{H}(11) \cdots \mathrm{O}(6)^{1}$ & 0.93 & 2.63 & $3.453(3)$ & 147.3 \\
\hline $\mathrm{C}(14)-\mathrm{H}(14) \cdots \mathrm{O}(5)^{2}$ & 0.93 & 2.30 & $3.207(3)$ & 165.8 \\
\hline $\mathrm{C}\left(24 \mathrm{~A}^{\wedge} b\right)-\mathrm{H}\left(24 \mathrm{D}^{\wedge} b\right) \cdots \mathrm{O}(5)^{3}$ & 0.97 & 2.45 & $3.111(16)$ & 125.3 \\
\hline $\mathrm{C}(7)-\mathrm{H}(7) \cdots \mathrm{O}\left(8^{\wedge} \mathrm{a}\right)^{4}$ & 0.93 & 2.51 & $3.258(12)$ & 137.9 \\
\hline $\mathrm{O}\left(8 \mathrm{~A}^{\wedge} \mathrm{b}\right)-\mathrm{H}\left(8 \mathrm{AA}^{\wedge} \mathrm{b}\right) \cdots \mathrm{N}^{\prime}\left(4 \mathrm{~A}^{\wedge} \mathrm{b}\right)^{5}$ & 1.05 & 2.19 & $3.06(4)$ & 139.6 \\
\hline
\end{tabular}

Symmetry transformations used to generate equivalent atoms: ${ }^{1} \mathrm{x}, \mathrm{y}-1, \mathrm{z}{ }^{2}-\mathrm{x},-\mathrm{y}+1,-\mathrm{z}+1 ;{ }^{3} \mathrm{x}+1, \mathrm{y}, \mathrm{z} ;{ }^{4}-\mathrm{x}+$ $1,-\mathrm{y},-\mathrm{z}+1 ;^{5}-\mathrm{x}+1,-\mathrm{y},-\mathrm{z}$.

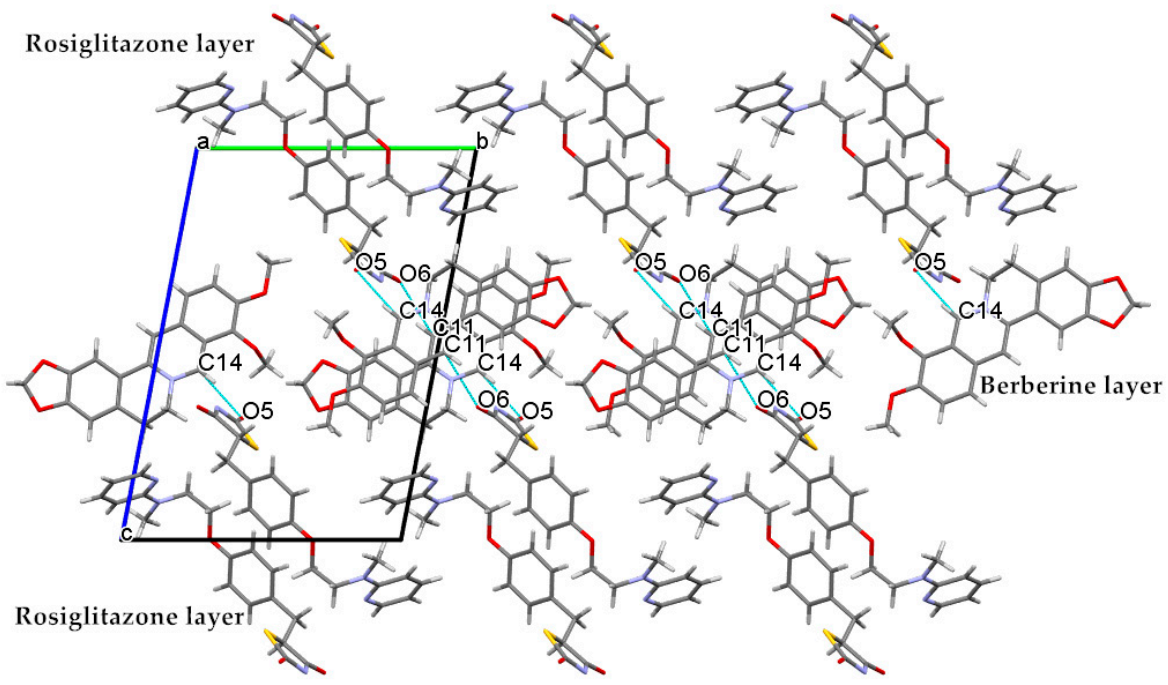

Figure 5. The unit cell diagram of Rsg-Bbr viewed along a-axis. Dashed lines represent hydrogenbonding interactions between Rsg and Bbr.

When the crystal structure elements were repeatedly arranged in three-dimensional space to form a co-crystal, a layer structure was generated in the structure, and $\pi$ electron interacted between layers. Unfolded the unit cell diagram, we found that the $\pi-\pi$ effect existed between the molecular layers. In Figure 6, there existed various $\pi-\pi$ interactions between benzene rings $\mathrm{A}$ and 
$\mathrm{C}$ of berberine. Berberine cation simultaneously interacted with two adjacent berberine through self-complementary $\pi-\pi$ interactions between rings $A$ and $C$. The distance of centroid $\cdots$ centroid was $3.854 \AA$ and $3.759 \AA$, respectively. A one-dimensional supramolecular layer of berberine resulted from the $\pi-\pi$ interactions. Rosiglitazone contained two non-coplanar A-phenyl ring and B-piperidine ring in its molecular structure, and there existed various $\pi-\pi$ interactions between rosiglitazone molecules. Ring A of rosiglitazone was involved in $\pi-\pi$ interactions with ring A of an adjacent rosiglitazone in the self-complementary way with a distance of $3.6604 \AA$ (centroid …centroid). Two adjacent rings B were also $\pi-\pi$ stacked in a self-complementary manner, with a distance of $3.5839 \AA$. These $\pi-\pi$ interactions combined with the $\mathrm{C}\left(24 \mathrm{~A}^{\wedge} \mathrm{b}\right)-\mathrm{H}\left(24 \mathrm{D}^{\wedge} \mathrm{b}\right) \cdots \mathrm{O}(5)$ between the rosiglitazone molecules to form a two-dimensional rosiglitazone layer (Figure 7). As a result, with non-covalent bonding forces such as hydrogen bonding, electrostatic attraction, and $\pi-\pi$ interaction, the crystal packing structure of Rsg-Bbr was formed.

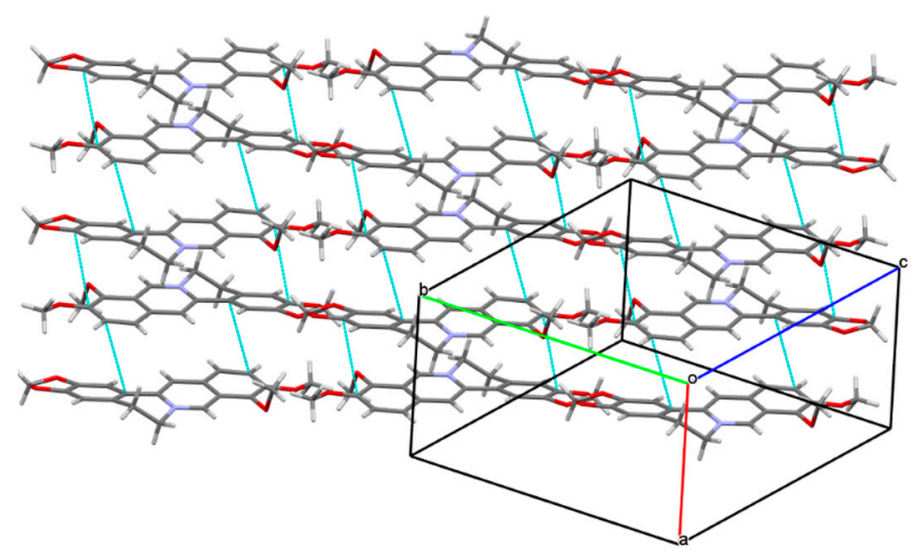

Figure 6. One-dimensional supramolecular layer Bbr resulting from $\pi-\pi$ interactions.

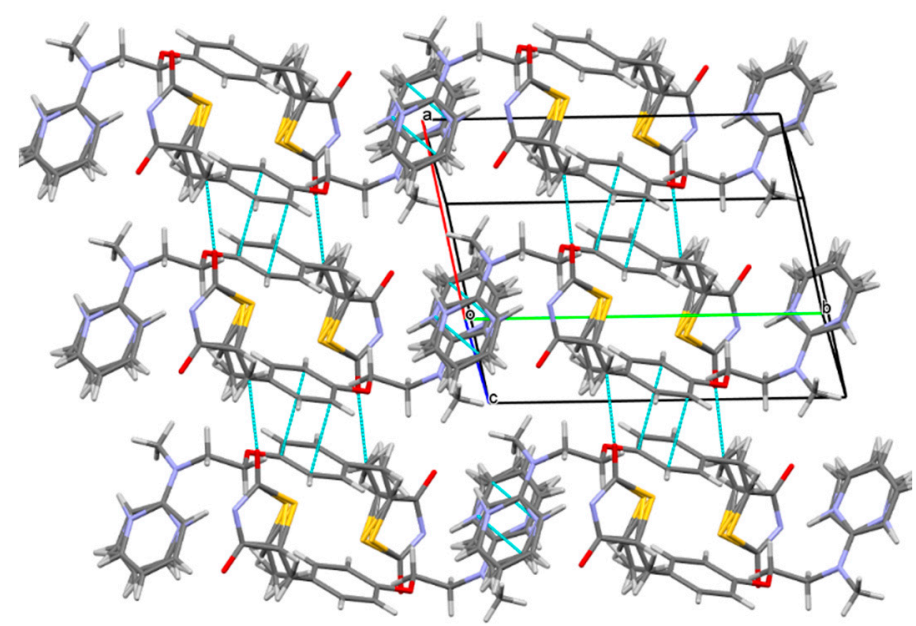

Figure 7. Two-dimensional supramolecular layer Rsg resulting from $\pi-\pi$ and C-H $\cdots$ O interactions.

Thermal analysis is often used to characterize the structure and stability of materials. Thermogravimetric analysis (TGA) and differential scanning calorimetry (DSC) were employed to investigate the thermal properties of Rsg-Bbr, Rsg, and Bbr samples. As shown in Figure 8a, the melting point of this co-crystal was between Rsg and Bbr, which their exothermic peaks accompanied by chemical decomposition at 172,157 , and $193{ }^{\circ} \mathrm{C}$, respectively. Furthermore, the TGA thermogram of Rsg-Bbr (Figure 8b) showed a slight but continuous weight loss of $4.42 \%$ before its melting point. This indicated the slow elimination of methanol molecules from the holes formed between the molecular layers. What's more, the weight of Rsg-Bbr did not change in the temperature range 120 to $210{ }^{\circ} \mathrm{C}$. Consequently, we can conclude that the substance has thermal stability in $120-210{ }^{\circ} \mathrm{C}$. 

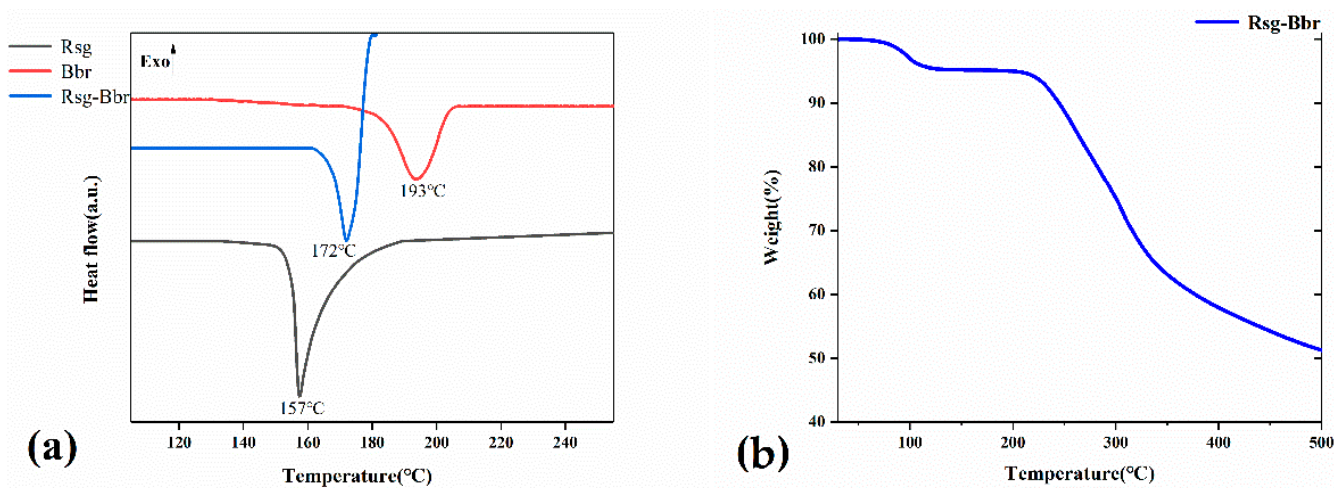

Figure 8. (a) DSC thermograms of Rsg, Bbr and Rsg-Bbr, respectively. (b) TG thermogram of Rsg-Bbr.

To observe the changes in the morphology of crystal, a polarizing optical microscope (POM) was used to observe the production of Rsg, Bbr and Rsg-Bbr. Each sample was observed at a magnification of 40x. It was obvious that the shape of Rsg-Bbr crystal was distinguishable from the two raw materials. This test further confirmed the presence of Rsg-Bbr adduct (Figure 9).

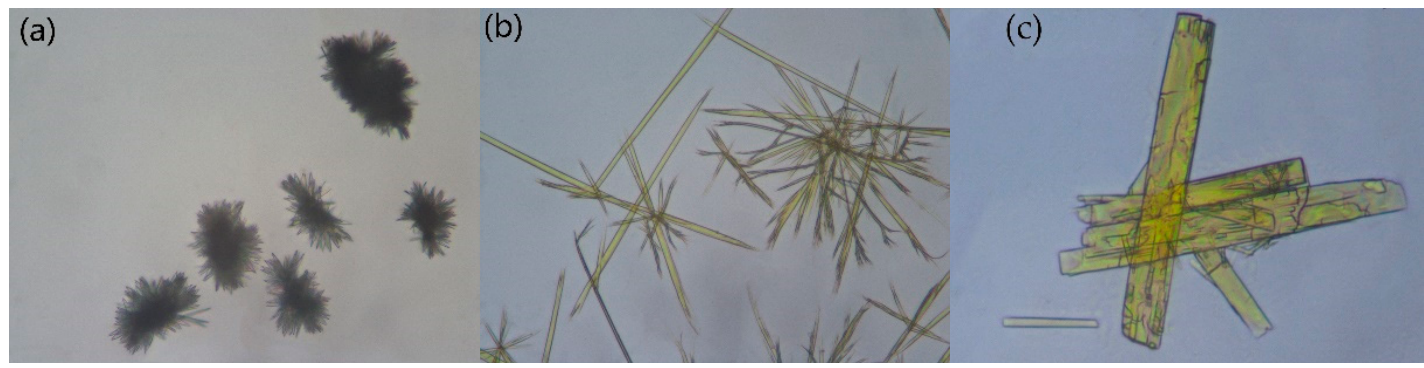

Figure 9. Micrographs of samples obtained by POM at $25^{\circ} \mathrm{C}$. (a) The crystal form of Rsg is rod-shaped (b) The crystal form of Bbr is needle-shaped (c) The crystal form of Rsg-Bbr is prismatic-shaped.

\subsection{The Characterization Analysis of Crystal Dissolution and Hygroscopicity}

Dissolution rate is one of the key pharmaceutical properties that need to be considered for successful drug delivery. Taking into account that the pKa of rosiglitazone is about 7 , which is a weakly basic drug and assimilated mainly in the intestine of the human body. Therefore, the phosphate buffer solution with a $\mathrm{pH}$ of 6.8 was used as the dissolution medium. According to Figure 10a below, the Rsg-Bbr group reported significantly and consistently higher dissolution than Rsg alone during the whole period. In the end, it peaked at the highest point of around 59\%. Due to the poor solubility of Rsg, its dissolution rate finally was less than $10 \%$. Notably, we also compared the dissolution rates of berberine and Rsg-Bbr. The dissolution rate of Bbr was significantly higher than that of the Rsg-Bbr in Figure 10b. Their highest value of rates achieved about $73 \%$ and $51 \%$ respectively. Compared the rate values of these samples, the trend depicting the dissolution rates of them was as follows: Bbr $>$ Rsg-Bbr $>$ Rsg. 
(a)

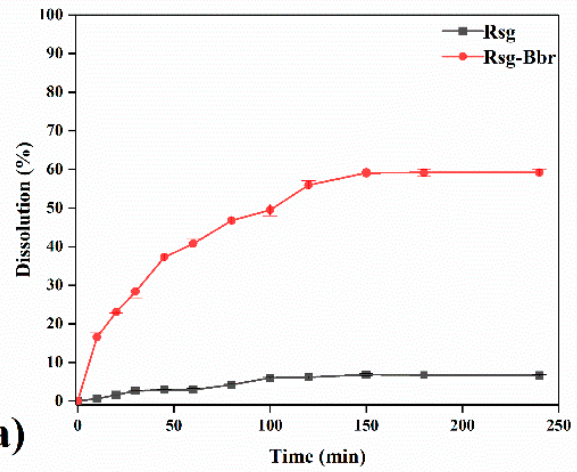

(b)

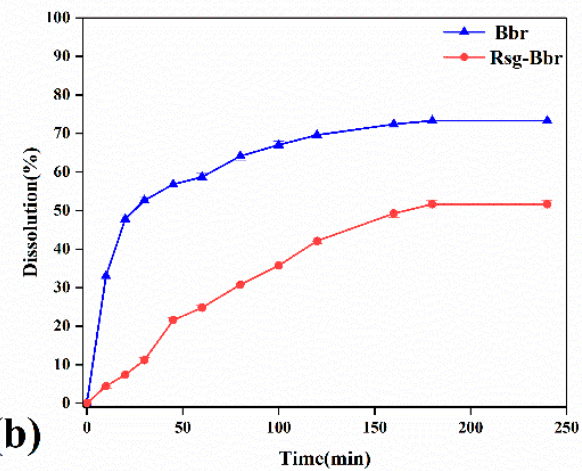

Figure 10. (a)The dissolution rate of Rsg and Rsg in the co-crystal; (b)The dissolution rate Bbr and Bbr in the co-crystal, respectively $(\mathrm{pH}=6.8)$.

Dynamic vapor sorption (DVS) analysis was performed to compare the hygroscopicity of Rsg-Bbr with Rsg. The sensitivity of a drug to moisture is one of the factors that affect its drug properties. The medicinal efficacy is affected badly by the moisture. According to reports, the berberine chloride has a hygroscopicity close to $20 \%$ in a high humidity environment with a relative humidity of $95 \%$, which is a medium hygroscopic substance [24]. As exhibited in Figure 11a, Rsg did not begin to absorb $0.46 \%$ water until the relative humidity reached $70 \%$. This result was expected because Rsg has poor solubility and hence low hygroscopicity. In Figure $11 \mathrm{~b}$, Rsg-Bbr presented low moisture and $0.56 \%$ of water in the range of $0-95 \% \mathrm{RH}$ at $25{ }^{\circ} \mathrm{C}$. This indicated that the co-crystal was non-hygroscopic. In addition, the desorption curve was close to the absorption curve for Rsg-Bbr, indicating the absorption and desorption processes of the co-crystal was reversible [10].

(a)

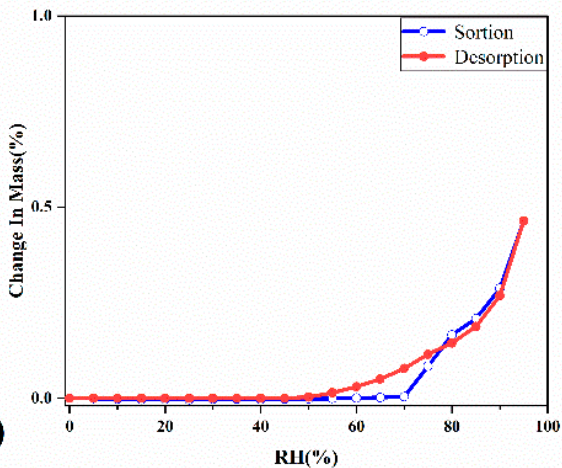

(b)

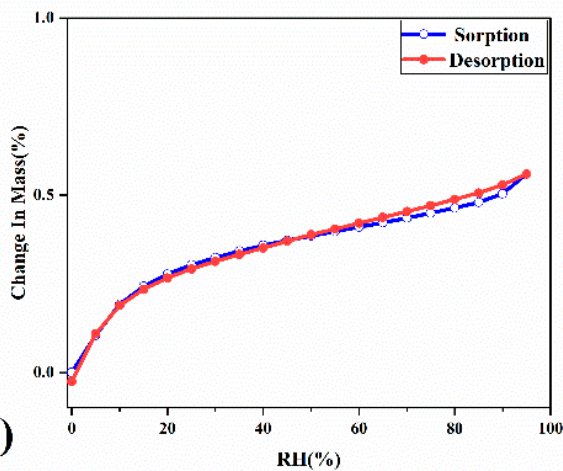

Figure 11. (a) DVS isotherm plots for co-crystal of Rsg-Bbr; (b) DVS isotherm plots for raw material of Rsg at $25^{\circ} \mathrm{C}$.

\section{Materials and Methods}

\subsection{Materials}

Rosiglitazone was purchased from Adamas Reagent Company (Shanghai, China). Berberine chloride (West plant extraction factory, Sichuan, China) was used as received. Other chemicals were purchased from Adamas Reagent Company and used without any further purification.

\subsection{Methods of Synthesis}

Synthesis of Rosiglitazone Sodium: Sodium metal $(0.07 \mathrm{~g}, 0.003 \mathrm{~mol})$ was shredded and added to $15 \mathrm{~mL}$ of anhydrous ethanol to make be sodium ethoxide. Rsg $(1.07 \mathrm{~g}, 0.003 \mathrm{~mol})$ was dissolved in $80 \mathrm{~mL}$ of ethanol by heating at $70^{\circ} \mathrm{C}$. Pouring into sodium ethoxide after the solution of Rsg was clarified. Then, the whole reaction system refluxed for $10 \mathrm{~h}$. Finally, filtered the suspension to get the white powdery rosiglitazone sodium. 
Synthesis of Rsg-Bbr: Berberine chloride $(1.17 \mathrm{~g}, 0.003 \mathrm{~mol})$ and the prepared sodium rosiglitazone salt were input $60 \mathrm{~mL}$ of methanol to dissolve. Then, heated to $70^{\circ} \mathrm{C}$ and refluxed for $1 \mathrm{~h}$. The obtained yellow filtrate was placed at $4{ }^{\circ} \mathrm{C}$. Crystals suitable for single crystal $\mathrm{X}$-ray diffraction experiment was obtained in 5 days.

\subsection{Methods of Structural Analysis}

\subsubsection{Fourier Transformation Infrared Spectroscopy (FT-IR)}

FT-IR spectra were collected by a Nicolet Spectrum FT-IR spectrometer (Nicolet iS10, Waltham, MA, USA) in the range from 4000 to $500 \mathrm{~cm}^{-1}$, with a resolution of $4 \mathrm{~cm}^{-1}$ at ambient conditions. Each compound ( $1 \mathrm{mg}$ ) was ground into the powder with dried $\mathrm{KBr}(50 \mathrm{mg})$ in a mortar and the mixture was pressed into a piece of slice.

\subsubsection{Powder X-ray Diffraction (PXRD)}

Powders were analyzed on a Bruker D8 Advance X-ray diffractometer (Bruker, Karlsruhe, Germany) with $\mathrm{Cu} \mathrm{K} \alpha$ radiation (1.54056 ̊), with two-theta pre-calibrated using a silicon standard. Data for each sample were collected from 5 to $50^{\circ}$ two theta with a step size of $0.02^{\circ}$ at $1 \mathrm{~s} /$ step and at $25{ }^{\circ} \mathrm{C}$ with step and scan speed of $5^{\circ} / \mathrm{min}$. The tube voltage and amperage were at $40 \mathrm{kV}$ and $100 \mathrm{~mA}$, respectively.

\subsubsection{Thermal Gravimetric Analysis (TGA)}

Samples (10 mg) were heated in a hermetically sealed aluminum pan containing a pinhole, on a NETZSCH STA $449 \mathrm{C}$ (NETZSCH, Selb, Germany) from room temperature to $500{ }^{\circ} \mathrm{C}$ at $10{ }^{\circ} \mathrm{C} / \mathrm{min}$ under $50 \mathrm{~mL} / \mathrm{min}$ dry nitrogen purge.

\subsubsection{Differential Scanning Calorimetry (DSC)}

Differential scanning calorimetry can detect the inherent melting point of each crystal. Powder samples $(5 \mathrm{mg})$ were heated from 25 to $250{ }^{\circ} \mathrm{C}$ with a heating rate of $10^{\circ} \mathrm{C} / \mathrm{min}$ on a NETZSCH-TA4 STA Instruments $449 \mathrm{C}$ differential scanning calorimeter (NETZSCH, Selb, Germany) under a continuously purged dry nitrogen atmosphere (flow rate of $50 \mathrm{~mL} / \mathrm{min}$ ). Tzero hermetic sealed aluminum pans were used for all samples. The instrument was pre-calibrated for temperature and enthalpy using indium.

\subsubsection{Single Crystal X-ray Diffraction (SCXRD)}

Single crystal X-ray diffraction was carried out on a SMART CCD diffractometer (Bruker, Karlsruhe, Germany). The data collection was performed at $293 \mathrm{~K}$ using $\mathrm{CuK} \alpha$ radiation $(\lambda=1.54184 \AA$, graphite monochromator). The integrated and scaled data were empirically corrected for absorption effects with spherical harmonics, implemented with the Sadabs scaling algorithm. Using Olex2 [25], the structure was solved by the ShelXS [26] structure solution program using direct methods and refined with the ShelXL [27] refinement package using least squares minimization. All non-hydrogen atoms were refined with anisotropic displacement parameters. All hydrogen atoms were located from the difference Fourier map and allowed to ride on their parent atoms in the refinement cycles.

\subsubsection{Polarized Optical Microscopy (POM)}

Several droplets of Rsg, Bbr and Rsg-Bbr are deposited on the microscope slide and sliced for stably observed their crystal morphology. These samples were optically characterized at $25^{\circ} \mathrm{C}$ by POM using an Olympus transmission microscope coupled with a Leica digital camera and Leica Application Suite Software. 


\subsection{Methods of Physicochemical Properties}

\subsubsection{Dissolution Rate}

For Rsg, Bbr and the co-crystal, the dissolution rate was performed by using a US Pharmacopoeia tablet dissolution test apparatus (paddle method, Hanson Research, Chatsworth, CA, USA) in 900 mL of phosphate buffer ( $\mathrm{pH} 6.8)$ containing $0.2 \%(w / v)$ of tween-80 as a dissolution medium. The rotation speed was set at $50 \mathrm{rpm}$ with dissolution bath temperature of $37^{\circ} \mathrm{C}$. Pure Rsg, Bbr and Rsg-Bbr powder were added to dissolution vessel containing $900 \mathrm{~mL}$ of phosphate buffer $6.8 \mathrm{pH}$. Aliquots of the dissolution medium ( $5 \mathrm{~mL})$ were withdrawn at 10, 20, 30, 45, 60, 80, 100, 120, 160, 180 and $240 \mathrm{~min}$. The dissolution media was replaced with an equivalent volume of fresh media at $37^{\circ} \mathrm{C}$. $4 \mathrm{~mL}$ of the secondary filtrate was taken by filtering with $0.45 \mu \mathrm{m}$ filter (Titan, Shanghai, China) and discarding the primary filtrate. The UV-Visible detector (UV2600, Shimadzu, Kyoto, Japan) were set at two wavelengths of $235 \mathrm{~nm}$ and $345 \mathrm{~nm}$ (the wavelength of $235 \mathrm{~nm}$ to detect Rsg, the wavelength of $345 \mathrm{~nm}$ to detect $\mathrm{Bbr}$ ). Each sample is balanced for 3 times. At last but not least, the data were detected by UV-Visible detection and linear regression were observed in the concentration $(\mathrm{C}, \mu \mathrm{g} / \mathrm{mL})$ by peak area $(\mathrm{A})$ that equation were: $\mathrm{A}_{\mathrm{Rsg}}=0.0267 \mathrm{C}+0.0114(\mathrm{r}=0.9999)$ and $\mathrm{A}_{\mathrm{Bbr}}=0.018 \mathrm{C}+0.0861$ $(\mathrm{r}=0.9998)$. The value of the dissolution rate calculated by the equation $\mathrm{w}=\mathrm{CV} / \mathrm{m} \times 100 \%$ was used to draw, ultimately.

\subsubsection{Dynamic Water Vapor Sorption Isotherm (DVS)}

Water sorption and desorption profiles of the materials were measured by using an automated vapor sorption analyzer (SMS Ltd., London, UK) at $25^{\circ} \mathrm{C}$. Samples of Rsg-Bbr and Rsg were studied at each step with the equilibration criteria of either $\mathrm{dm} / \mathrm{dt} \leq 0.003 \%$ (the defining time is $5 \mathrm{~min}$ ) or maximum equilibration time of $6 \mathrm{~h}$. Once one of the criteria is met, the relative humanity $(\mathrm{RH}) \mathrm{was}$ changed to the next target value, following the $0 \%-95 \%-0 \%$ sorption and desorption cycle with the step size of $5 \%$ RH.

\section{Conclusions}

The co-crystal of rosiglitazone (Rsg) with berberine chloride (Bbr), Rsg-Bbr, was created and characterized. As far as we know, this is the first report to show a new drug-drug co-crystal formed by Rsg and a natural drug with a hypoglycemic effect. The preparation process was simple with easy handling. Crystal structure analysis showed that the nitrogen anion of rosiglitazone successfully has replaced the chloride ion in berberine chloride to form the co-crystal. The electrostatic attraction was also generated between the anion and cation. Moreover, this electrostatic interaction combined with the C-H...O hydrogen bond formed between Rsg and Bbr and the $\pi-\pi$ interaction formed between conjugated rings to dominate the crystal packing structure.

Additionally, Rsg-Bbr exhibits a low moisture adsorption curve in the range of 0-95\% relative humidity at $25{ }^{\circ} \mathrm{C}$. Rsg in the form of the co-crystal has a higher dissolution rate compared with pure rosiglitazone. The work described above shows a promising method that can be used to overcome the poor physicochemical properties of the parent drugs by forming the co-crystal structure. The pharmaceutical crystals based on drug-drug combinations could contribute to the development of new drugs in the pharmaceutical field.

Author Contributions: Data curation, X.G., L.C., and L.Z.; formal analysis, X.G., L.C. and L.Z.; funding acquisition, X.H. and L.J.; investigation, X.G. and L.J.; project administration, X.H. and L.J.; software, X.G. and L.J.; writing-original draft, X.G. All authors have read and agreed to the published version of the manuscript.

Funding: The authors acknowledge the financial support received from Chongqing Science and Technology Commission [cstc2019jscx-msxmX0096]. We also appreciate that the undergraduate everyone innovation program of school of pharmacy of Chongqing Medical University [DXSZCXM201905] provide partial financial aid for our work.

Conflicts of Interest: The authors declare no conflict of interest. 


\section{References}

1. Aakeroy, C.B.; Salmon, D.J. Building co-crystals with molecular sense and supramolecular sensibility. Crystengcomm 2005, 7, 439-448. [CrossRef]

2. Duggirala, N.K.; Perry, M.L.; Almarsson, O.; Zaworotko, M.J. Pharmaceutical cocrystals: Along the path to improved medicines. Chem. Commun. 2016, 52, 640-655. [CrossRef] [PubMed]

3. Sun, C.C. Cocrystallization for successful drug delivery. Expert Opin. Drug Deliv. 2013, 10, $201-213$. [CrossRef] [PubMed]

4. Drozd, K.V.; Manin, A.N.; Churakov, A.V.; Perlovich, G.L. Novel drug-drug cocrystals of carbamazepine with para-aminosalicylic acid: Screening, crystal structures and comparative study of carbamazepine cocrystal formation thermodynamics. Crystengcomm 2017, 19, 4273-4286. [CrossRef]

5. Jiang, L.; Huang, Y.; Zhang, Q.; He, H.; Xu, Y.; Mei, X. Preparation and Solid-State Characterization of Dapsone Drug-Drug Co-Crystals. Cryst. Growth Des. 2014, 14, 4562-4573. [CrossRef]

6. Kaur, R.; Cavanagh, K.L.; Rodriguez-Hornedo, N.; Matzger, A.J. Multidrug Cocrystal of Anticonvulsants: Influence of Strong Intermolecular Interactions on Physiochemical Properties. Cryst. Growth Des. 2017, 17, 5012-5016. [CrossRef]

7. Putra, O.D.; Furuish, T.; Yonemochi, E.; Terada, K.; Uekusa, H. Drug-Drug Multicomponent Crystals as an Effective Technique to Overcome Weaknesses in Parent Drugs. Cryst. Growth Des. 2016, 16, 3577-3581. [CrossRef]

8. Kaplinsky, E. Sacubitril/valsartan in heart failure: Latest evidence and place in therapy. Ther. Adv. Chronic Dis. 2016, 7, 278-290. [CrossRef]

9. Kario, K.; Sun, N.; Chiang, F.-T.; Supasyndh, O.; Baek, S.H.; Inubushi-Molessa, A.; Zhang, Y.; Gotou, H.; Lefkowitz, M.; Zhang, J. Efficacy and Safety of LCZ696, a First-in-Class Angiotensin Receptor Neprilysin Inhibitor, in Asian Patients With Hypertension. Hypertension 2014, 63, 698-705. [CrossRef]

10. Deng, Y.; Zhang, Y.; Huang, Y.; Zhang, M.; Lou, B. Preparation, Crystal Structures, and Oral Bioavailability of Two Cocrystals of Emodin with Berberine Chloride. Cryst. Growth Des. 2018, 18, 7481-7488. [CrossRef]

11. Kahn, B.B.; McGraw, T.E. Rosiglitazone, PPAR (gamma), and Type 2 Diabetes. N. Engl. J. Med. 2010, 363, 2667-2669. [CrossRef] [PubMed]

12. Lehmann, J.M.; Moore, L.B.; Smith-Oliver, T.A.; Wilkison, W.O.; Willson, T.M.; Kliewer, S.A. An antidiabetic thiazolidinedione is a high affinity ligand for peroxisome proliferator-activated receptor gamma (PPAR gamma). J. Biol. Chem. 1995, 270, 12953-12956. [CrossRef] [PubMed]

13. American Diabetes Association. Introduction: Standards of Medical Care in Diabetes-2019. Diabetes Care 2019, 42, S1-S2. [CrossRef]

14. Balfour, J.A.B.; Plosker, G.L. Rosiglitazone. Drugs 1999, 57, 921-930. [CrossRef]

15. Lan, J.; Zhao, Y.; Dong, F.; Yan, Z.; Zheng, W.; Fan, J.; Sun, G. Meta-analysis of the effect and safety of berberine in the treatment of type 2 diabetes mellitus, hyperlipemia and hypertension. J. Ethnopharmacol. 2015, 161, 69-81. [CrossRef]

16. Kuo, C.L.; Chi, C.W.; Liu, T.Y. The anti-inflammatory potential of berberine in vitro and in vivo. Cancer Lett. 2004, 203, 127-137. [CrossRef]

17. Pan, G.-Y.; Wang, G.-J.; Sun, J.-G.; Huang, Z.-j.; Zhao, X.-C.; Gu, Y.; Liu, X.-D. Inhibitory action of berberine on glucose absorption. Yaoxue Xuebao 2003, 38, 911-914.

18. Homma, N.; Kono, M.; Kadohira, H.; Yoshihara, S.; Masuda, S. The effect of berberine chloride on the intestinal flora of infants. Arzneim. Forsch. 1961, 11, 450-454.

19. Li, G.-X.; Wang, X.-m.; Jiang, T.; Gong, J.-f.; Nu, L.-y.; Li, N. Berberine Prevents Intestinal Mucosal Barrier Damage During Early Phase of Sepsis in Rat through the Toll-Like Receptors Signaling Pathway. Korean J. Physiol. Pharmacol. 2015, 19, 1-7. [CrossRef]

20. Zhang, H.; Wei, J.; Xue, R.; Wu, J.-D.; Zhao, W.; Wang, Z.-Z.; Wang, S.-K.; Zhou, Z.-X.; Song, D.-Q.; Wang, Y.-M.; et al. Berberine lowers blood glucose in type 2 diabetes mellitus patients through increasing insulin receptor expression. Metab. Clin. Exp. 2010, 59, 285-292. [CrossRef]

21. Chen, C.; Yu, Z.; Li, Y.; Fichna, J.; Storr, M. Effects of Berberine in the Gastrointestinal Tract-A Review of Actions and Therapeutic Implications. Am. J. Chin. Med. 2014, 42, 1053-1070. [CrossRef] 
22. Zhou, J.Y.; Zhou, S.W.; Bin Zhang, K.; Tang, J.L.; Guang, L.X.; Ying, Y.; Xu, Y.; Zhang, L.; Li, D.D. Chronic effects of berberine on blood, liver glucolipid metabolism and liver PPARs expression in diabetic hyperlipidemic rats. Biol. Pharm. Bull. 2008, 31, 1169-1176. [CrossRef]

23. Zhang, X.; Zhao, Y.; Xu, J.; Xue, Z.; Zhang, M.; Pang, X.; Zhang, X.; Zhao, L. Modulation of gut microbiota by berberine and metformin during the treatment of high-fat diet-induced obesity in rats. Sci. Rep. 2015, 5. [CrossRef] [PubMed]

24. Wang, C.; Perumalla, S.R.; Lu, R.; Fang, J.; Sun, C.C. Sweet Berberine. Cryst. Growth Des. 2016, 16, $933-939$. [CrossRef]

25. Dolomanov, O.V.; Bourhis, L.J.; Gildea, R.J.; Howard, J.A.K.; Puschmann, H. OLEX2: A complete structure solution, refinement and analysis program. J. Appl. Crystallogr. 2009, 42, 339-341. [CrossRef]

26. Sheldrick, G.M. A short history of SHELX. Acta Crystallogr. A-Found. Adv. 2008, 64, 112-122. [CrossRef] [PubMed]

27. Sheldrick, G.M. Crystal structure refinement with SHELXL. Acta Crystallogr. Sect. C-Struct. Chem. 2015, 71, 3-8. [CrossRef]

Sample Availability: Samples of the compounds are not available from the authors.

(C) 2020 by the authors. Licensee MDPI, Basel, Switzerland. This article is an open access article distributed under the terms and conditions of the Creative Commons Attribution (CC BY) license (http://creativecommons.org/licenses/by/4.0/). 